\title{
IoMT-Based Automated Detection and Classification of Leukemia Using Deep Learning
}

\author{
Nighat Bibi $\mathbb{D}^{1},{ }^{1}$ Misba Sikandar $\left(\mathbb{D},{ }^{1}\right.$ Ikram Ud Din $\left(\mathbb{D},{ }^{1}\right.$ Ahmad Almogren $\left(\mathbb{D},{ }^{2}\right.$ \\ and Sikandar Ali $\left.{ }^{3}\right)^{3}$ \\ ${ }^{1}$ Department of Information Technology, TheUniversity of Haripur, Haripur 22620, Pakistan \\ ${ }^{2}$ Department of Computer Science, College of Computer and Information Sciences, King Saud University, Riyadh 11633, \\ Saudi Arabia \\ ${ }^{3}$ Beijing Key Laboratory of Petroleum Data Mining, China University of Petroleum-Beijing, Beijing 102249, China
}

Correspondence should be addressed to Sikandar Ali; sikandar@cup.edu.cn

Received 22 October 2020; Revised 2 November 2020; Accepted 21 November 2020; Published 4 December 2020

Academic Editor: Shah Nazir

Copyright (c) 2020 Nighat Bibi et al. This is an open access article distributed under the Creative Commons Attribution License, which permits unrestricted use, distribution, and reproduction in any medium, provided the original work is properly cited.

\begin{abstract}
For the last few years, computer-aided diagnosis (CAD) has been increasing rapidly. Numerous machine learning algorithms have been developed to identify different diseases, e.g., leukemia. Leukemia is a white blood cells- (WBC-) related illness affecting the bone marrow and/or blood. A quick, safe, and accurate early-stage diagnosis of leukemia plays a key role in curing and saving patients' lives. Based on developments, leukemia consists of two primary forms, i.e., acute and chronic leukemia. Each form can be subcategorized as myeloid and lymphoid. There are, therefore, four leukemia subtypes. Various approaches have been developed to identify leukemia with respect to its subtypes. However, in terms of effectiveness, learning process, and performance, these methods require improvements. This study provides an Internet of Medical Things- (IoMT-) based framework to enhance and provide a quick and safe identification of leukemia. In the proposed IoMT system, with the help of cloud computing, clinical gadgets are linked to network resources. The system allows real-time coordination for testing, diagnosis, and treatment of leukemia among patients and healthcare professionals, which may save both time and efforts of patients and clinicians. Moreover, the presented framework is also helpful for resolving the problems of patients with critical condition in pandemics such as COVID-19. The methods used for the identification of leukemia subtypes in the suggested framework are Dense Convolutional Neural Network (DenseNet-121) and Residual Convolutional Neural Network (ResNet-34). Two publicly available datasets for leukemia, i.e., ALL-IDB and ASH image bank, are used in this study. The results demonstrated that the suggested models supersede the other well-known machine learning algorithms used for healthy-versus-leukemia-subtypes identification.
\end{abstract}

\section{Introduction}

Internet of Things (IoT) is deployed in several areas, such as vehicular communications [1,2], smart cites [3], cloud computing $[4,5]$, smart ecosystems $[6,7]$, smart campus [8], mobile communications [9], smart agriculture [10], and Healthcare or Internet of Medical Things (IoMT) [11]. However, a large section of the research community is attracted by IoMT or simply Healthcare IoT. IoMT $[12,13]$ is indeed a set of WiFi smart medical gadgets and smart applications connected to IT health systems through computer networks [14-16]. Smart medical devices are equipped with sensors or other computing resources and are exclusively intended for healthcare in the body at home, clinic, hospital, and community $[17,18]$. These smart devices are linked to the cloud platforms to analyze collected data for further processing [19]. The IoMT technology includes virtual care for patients with long-term illnesses, portable mHealth devices for patients, monitoring of patients' medication, tracking the location of hospitalized patients $[20,21]$, and the ability to provide information to caregivers $[22,23]$. The IoMT technology saves time and efforts of patients and doctors [11]. Connecting patients to their doctors and enabling the transfer of medical data over a secure network reduce the burden on health systems [24]. A rapid increase in the development and use of IoMTopens the door to deploying such frameworks that can fastly, securely, and accurately examine the patients' health and 
diagnose and cure different diseases remotely [25]. IoMTbased frameworks are abundant, particularly for those diseases which are more crucial with respect to patients' life, such as leukemia.

1.1. Leukemia. Leukemia is a disease related to white blood cells (WBC). Platelets, red blood cells (RBC), and WBC are various components of blood. Platelets help to clot and control bleeding. RBC known as erythrocytes are responsible for the transfer of oxygen through lungs to the body tissues. While WBC, also known as leukocytes, are responsible for fighting against diseases and infections. Leukemia refers to the production of large numbers of immature WBC. It is a type of cancer that affects the bone marrow and blood while destroying the immune system of a human body. Two main categories of leukemia based on progress are acute and chronic leukemia. Infected WBC grow rapidly in acute leukemia and do not perform in a normal way, whereas in chronic leukemia, WBC can act normally and grow less quickly. However, this can be severe since it may not be distinguished easily from the normal WBC. In addition, two types of acute and chronic leukemia are lymphoid and myeloid leukemia based on the size and shape of WBC, where both of them can be further divided into two subtypes each, i.e., acute lymphocytic leukemia (ALL), chronic lymphocytic leukemia (CLL), acute myeloid leukemia (AML), and chronic myeloid leukemia (CML).

1.1.1. Acute Lymphocytic Leukemia. ALL is mostly seen in children, which is a WBC cancer caused by the constant multiplication and overproduction of immature WBC in the bone marrow. The symptoms of ALL are quite similar to flu and other common diseases, such as exhaustion, weakness, and pain in joints and bones, making it very difficult to diagnose this disease. Three types of ALL are classified as L1, L2, and L3 [26].

1.1.2. Acute Myeloid Leukemia. The most common type of acute leukemia is AML, which happens when the bone marrow starts producing blasts and immature WBC. It may also create $\mathrm{RBC}$ and platelets that are abnormal. The common symptoms of early-stage AML may be similar to those of influenza or other common illnesses. Based on the types of blood cell affected, signs and symptoms may vary. The signs of AML are fever, bone pain, tiredness and fatigue, shortness of breath, pale skin, frequent infections, easy bruising, and abnormal bleeding, such as frequent nosebleeds and gum bleeding. AML has eight different subtypes that differentiate it from the other types of leukemia [27].

1.1.3. Chronic Lymphoblastic Leukemia. CLL is a hematological sickness that gets worse slowly. It is commonly observed in adults and is very uncommon in children. The symptoms of CLL include loss in weight, fever, night sweats, and periodic infections.

1.1.4. Chronic Myeloid Leukemia. CML, also known as chronic myelogenous leukemia, is a form of slow growing leukemia, but it can develop into acute leukemia, which is fast growing and difficult to treat. This may be viewed in three stages, i.e., chronic, accelerated, and blast stages. In the chronic stage, the leukemia is inside the strongest situation and grows slowly. Within the second stage, the blood cells are immature, usually referred to as extended stage. The third stage is the blast stage, which is also known as the acute stage or blast transformation stage. The pictorial representation of blood structure and leukemia types is shown in Figure 1.

It is necessary for hematologists to identify the existence of leukemia along with its particular form in order to avoid medical risks and to determine the correct leukemia treatment. A crucial and time-consuming step is the identification of leukemia through optical blood smears examination monitored by a specialist. To solve such problems, many CAD methodologies for quantitative analysis of the peripheral blood samples have been developed using machine learning and deep learning methods. However, these approaches have some drawbacks and need improvements in terms of accuracy, learning process, and efficiency.

Thus, to tackle these drawbacks and by keeping in view the vitality of healthcare, an IoMT-based framework for the automatic identification of leukemia subtypes is proposed in this study. In the proposed framework, IoT-enabled microscope uploads the blood smear images to the leukemia cloud. The leukemia with respect to its type(s) is diagnosed by using the deep learning models, i.e., ResNet-34 [28] or DenseNet-121 [29]. Deep learning is a branch of machine learning used to solve a variety of problems and describes the abstract concepts through different layers of data processing to discover better learning algorithms and representations that are less dependent on feature engineering [30]. The high prediction power of deep learning algorithms and surprising ability of features extraction extend their use to a wide range of research areas. Therefore, in this study, deep transfer learning-based methods are proposed to identify microscopic blood images as healthy, ALL, AML, CLL, and CML. The detail of the models is given in Section 3. The diagnostic results of leukemia with respect to its types predicted by the suggested models can be shown on the clinician's computer and accordingly, medical care may be offered to leukemia patients. The proposed framework is demonstrated in Figure 2.

The suggested framework is also helpful for patients and doctors in pandemics such as coronavirus disease 2019 (COVID-19). Due to the spread of COVID-19, most of the countries imposed sudden lockdown in major cities; as a result, almost ten billion citizens were quarantined. During this pandemic, people put their focus on accumulating more necessary care. However, some patients with chronic diseases leave their homes for medical help opening a gap in 


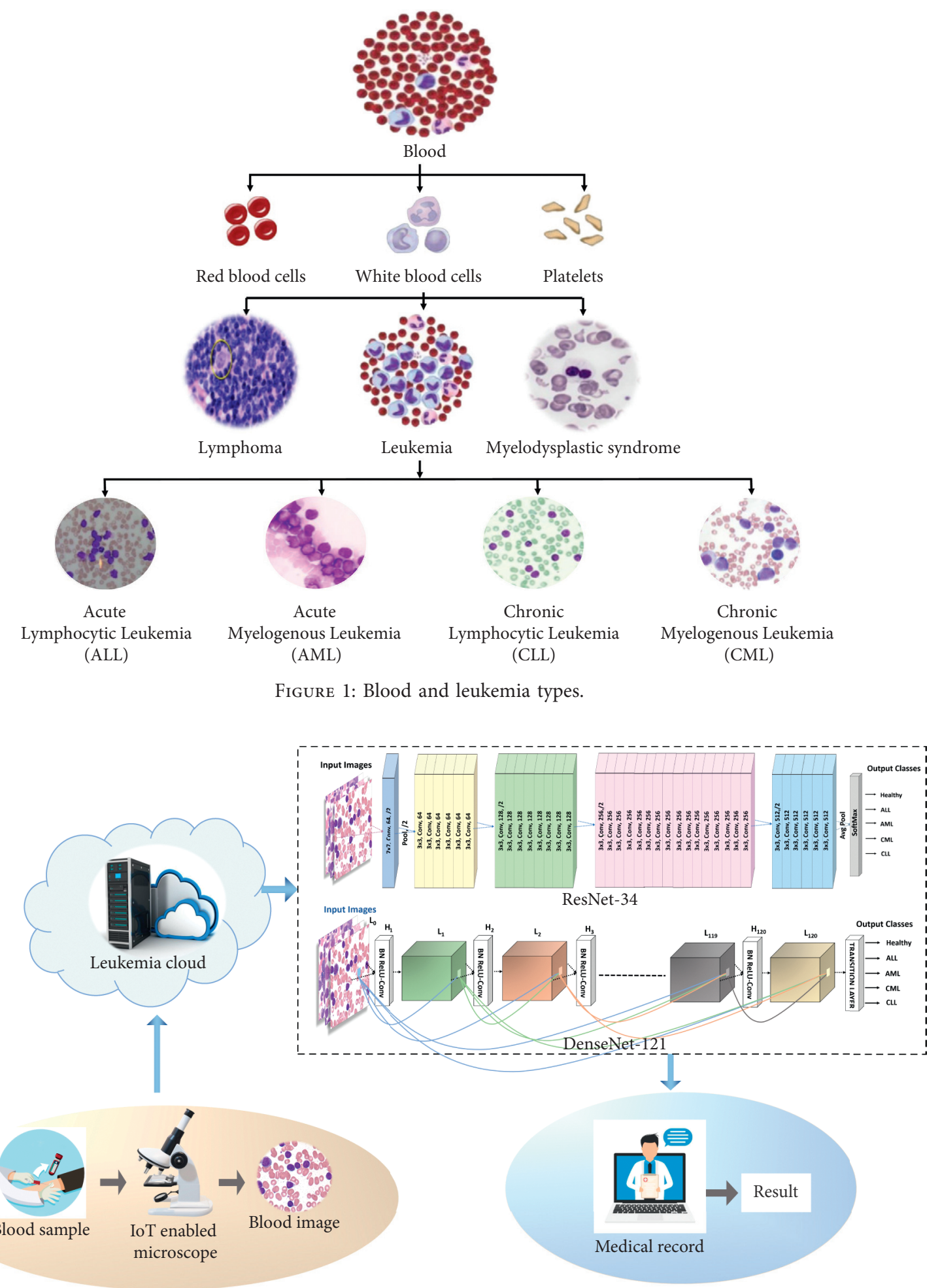

FIgURE 2: Proposed framework for automated leukemia diagnosis.

quarantine measures, which is a threat to disease control. Hence, if the proposed framework is applied to the current crisis, it would provide a medical platform that may help patients to receive adequate medical care at homes. The proposed approach is fast and accurate in addition to reducing the need for an expert oncologist to diagnose leukemia or any of its subtypes.

We delineate our experiments in the following sections. The related work is presented in Section 2. Section 3 elaborates the data exploration, augmentation, and the proposed 
models. Section 4 describes the experimental results, while Section 5 concludes the conducted study and presents future research directions.

\section{Literature Survey}

For the computer-aided diagnosis (CAD) of leukemia, various studies have been conducted by the IoMT research community. These studies present different machine and deep learning algorithms for the identification of leukemia. In [31], the classification and detection of WBC cancer and some of its subtypes are done by using Random Forest with $94.3 \%$ accuracy. In [32], the proposed model detected ALL using KNN and Naive Bayes Classifier with 92.8\% accuracy. The classifier is tested on 60 sample images. In [33], a novel Principal Component Analysis (PCA) based on ABC-BPNN scheme is suggested for the classification of leukemia cells and attained $98.72 \%$ average accuracy with reduction in the computation time. In [34], the ALL is identified. Firstly, leukemia images are segmented by BSA-based clustering. Secondly, Jaya technique is applied in integration with some standard classification methods such as Naive Bayes, K-nearest neighbor, linear discriminant analysis, support vector machine (SVM), ensemble random under-sampling boost, and decision tree. However, Jaya with SVM and Jaya with decision tree gave better accuracy.

The Linear Discriminant Analysis- (LDA-) based PCA model for diagnosing leukemia by utilizing Discrete Orthogonal Stockwell Transform (DOST) method to extract the features of microscopic images is presented in [35]. In [36], for feature extraction, three pre-trained CNN architectures are applied. However, for the leukemia classification on the hybrid database, SVM without segmentation is used. In [37], for the identification of acute leukemia from microscopic images initially to extract features, the images were segmented by using unsharp masking sub-imaging bounding box and $\mathrm{L} \times \mathrm{a} \times \mathrm{b}$ color Fuzzy C-Means Clustering after applying the Nearest Neighbor. Then, to identify ALL, the extracted features were passed to SVM, which attained 95\% accuracy. In [38], a robust feature extraction and selection technique for the identification of lymphocytes versus ALL is proposed. The classification is done by KNN using Euclidean Distance with 92.5\% accuracy. In [39], the classification is done using CNN, where only ALL vs healthy samples are classified with $88.25 \%$ accuracy and leukemia subtypes are classified with $81.74 \%$ accuracy.

In $[35,40-43]$, ALL-IDB dataset is used to detect ALL. In [40], K-medoids is presented with $98.60 \%$ accuracy. In [35], DOST, PCA, and LDA are proposed with 99.66\% accuracy. In [41], Generative Adversarial Optimization- (GAO-) based method is investigated with $93.84 \%$ accuracy. In [42], Genetic Algorithm (GA) and Artificial Neural Network (ANN) are presented with $97.07 \%$ accuracy. In [43], Chronological Sine Cosine Algorithm (SCA) is tested, which achieved 98.70\% accuracy. In [44], ASH image bank and ALL-IDB1 datasets are utilized for classifying lymphoblast cells. Convnet is investigated and achieved $81.74 \%$ accuracy. In [36], heterogeneous database ALL-IDB1 and ALL-IDB2 datasets are used. The diagnosis of leukemia (pathological or nonpathological) is done using pre-trained CNN with SVM, which attained 99\% accuracy. In [45], the ALL-IDB1 dataset is employed where the diagnosis of leukemia (normal vs abnormal) is performed by CNN and achieved $96.60 \%$ accuracy.

In [46], the ALL-IDB1 and ALL-IDB2 datasets are employed for the ALL detection. However, the scheme used is SVM, which attained $89.81 \%$ accuracy. In [47], the ALL-IDB2 dataset is used for the identification of ALL where the method investigated is customized KNN with 96.25\% accuracy. In [48], by utilizing ALL-IDB1 dataset, ALL is classified on the basis of cell energy features by employing SVM, which attained $94.00 \%$ accuracy. In [49], by utilizing ASH image bank dataset, firstly FAB ALL subtypes are identified by applying GA with multilayer perceptron kernel (MLP) function and acquired 97.1\% accuracy. Secondly, FAB AML subtypes are identified by employing genetic phenomena with Gaussian radial basis kernel function and achieved $98.5 \%$ accuracy. Finally, healthy, ALL, and AML are identified by using a GA with Gaussian Radial Basis kernel and achieved $99.50 \%$ accuracy.

It is exhibited from the literature that almost all previous approaches identified leukemia with respect to healthy, AML or ALL types. However, these approaches did not address the problem of identifying leukemia with respect to all its subtypes, i.e., ALL, AML, CLL, and CML. Therefore, in this study, the deep CNN-based approaches are presented to classify leukemia in terms of all its types.

The proposed work is an advancement of the study conducted in [39], where the authors have done the classification of ALL vs. healthy using a simple CNN architecture. However, our proposed work focuses on the classification of four subtypes of leukemia, i.e., ALL, AML, CLL, and CML, using advanced CNN architectures-Residual Network-34 (ResNet-34) [28] and Dense Convolutional Network-121 (DenseNet-121) [29]. Hence, it is elaborated in the result section that the proposed deep CNNs, i.e., DenseNet-121 and ResNet-34, outperform the existing schemes with $99.91 \%$ and $99.96 \%$ accuracy, respectively. Furthermore, for investigating the proposed methods, ALL-IDB and ASH image bank datasets are utilized.

\section{Experimental Setup}

This section delineates the experimental setup for conducting the study. Firstly, the dataset retrieval is described followed by data augmentation, while in the end, the deep learning models used to classify the subtypes of leukemia are explained. The proposed methodology is presented in Figure 3.

3.1. Leukemia Database Description. The dataset is collected from two different sources: ASH image bank [50] and ALLIDB $[51,52]$. The ASH image bank is freely accessible on the Internet and contains a complete bank of images on a variety 




Figure 3: Proposed methodology.

of hematological topics. In this article, all accessible annotated cell images with leukemia of blood are selected including any one of the four subtypes.

The ALL-IDB dataset provides annotated microscopic images of blood cells that were developed for segmentation, evaluation, and classification. ALL-IDB contains only healthy and ALL types of leukemia samples. The remaining mentioned leukemia subtypes are not available in the ALLIDB dataset. The ALL-IDB is considered more reliable because experienced oncologists provided the ALL classification for each image in the dataset. Some samples of images from datasets are shown in Figure 4.

3.2. Reducing Overfitting. Data augmentation methods are commonly used for maximizing the size of datasets and reducing overfitting, especially in the deep learning models. Various image transformation schemes, such as rotation, flipping, and shifting, have been used to obtain distinct images from the original image. They would have more generalization capabilities if machine learning and deep learning models were trained with the original images along with their sub-versions. In different image segmentation studies with CNNs, numerous methods of information replication have reduced the model error rate by giving better speculation. Here, a better assortment of microscopic blood cell images is provided by the retrieved datasets, but both datasets contain samples of limited numbers of leukemia subtypes. As the sample datasets are very limited for deep learning techniques, it can lead to overfitting. Thus, image transformation or data augmentation techniques are used to maximize the dataset; i.e., rotation, height shift, width shift, horizontal flip, zoom, and shearing, are applied. The sample size after applying the methods of image transformation is increased in both datasets. Exact number of samples per leukemia subtype before and after augmentation is shown in Table 1. After applying the data augmentation, the sample size reached 3277 and 2359 in the ASH image bank and ALL-IDB datasets, respectively.
3.3. Deep Learning Models. After performing augmentation on the dataset, it is passed on to CNNs - the deep learning models. Instead of using traditional machine learning techniques which are provided with hand-crafted features to learn and require time and effort, in this study, CNNs are utilized. CNNs have the ability to learn automatically from raw data. A typical $\mathrm{CNN}$ begins with convolutional and pooling layers and ends with a fully connected layer. New models can be created from a CNN by assembling some convolutional, pooling, and dense layers. By arranging some layers of CNNs architectures, new light CNN models have been developed such as VggNet [53] and AlexNet [54]. However, advanced CNN models, such as ResNet [28] and DenseNet [29], are deeper and more complex having the ability to learn better, as elaborated in the result section. Therefore, in the presented work, ResNet-34 and DenseNet121 models are used in a supervised learning mode to identify and categorize leukemia with respect to its types. The details of each model are described in the following subsections.

3.3.1. ResNet-34. ResNet-34 is a pre-trained 34-layer model. The performance of deep neural networks depends on architecture and dataset. A deep network of CNNs and large dataset produce better performance. However, the performance deteriorates after a certain depth when the network gets deeper. The reason of this problem is the vanishing gradient. The ResNet solves this problem as gradients flow from starting layers to the final ones by skipping some layers. Mathematically, the layers of the ResNet model can be calculated according to

$$
Y=f(x)+i d(x)=f(x)+x .
$$

By skipping the connections between layers, the gradient can easily flow and the training of the layers becomes faster. ResNet-34 consists of a total of 34 layers wherein one is convolutional and pooling layer in addition to four other layers with the same pattern. Each layer is convolved with $3 \times 3$ convolution with a feature map of sizes $64,128,256$, 


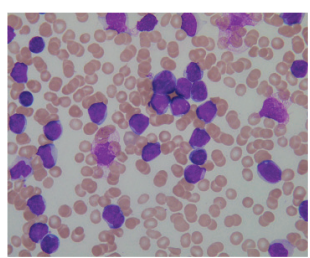

(a)

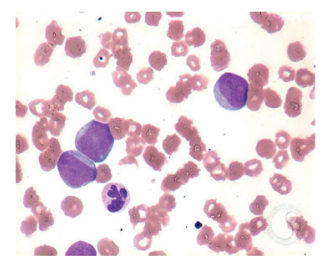

(b)

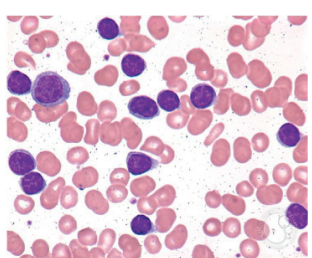

(c)

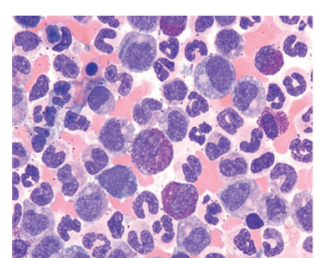

(d)

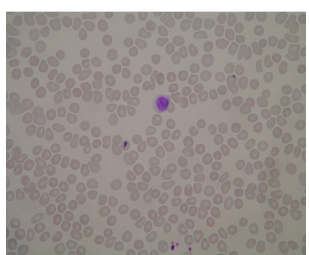

(e)

Figure 4: Leukemia subtype images. (a) Acute lymphocytic leukemia (ALL). (b) Acute myelogenous leukemia (AML). (c) Chronic lymphocytic leukemia (CLL). (d) Chronic myelogenous leukemia (CML). (e) Healthy.

TABLE 1: Distribution of leukemia subtypes before and after augmentation.

\begin{tabular}{lccc}
\hline Leukemia type & Dataset & Before augmentation & After augmentation \\
\hline ALL & ALL-IDB & 181 & 1079 \\
AML & ASH image bank & 55 & 1194 \\
CLL & ASH image bank & 38 & 840 \\
CML & ASH image bank & 57 & 1243 \\
Healthy & ALL-IDB & 187 & 1280 \\
\hline
\end{tabular}

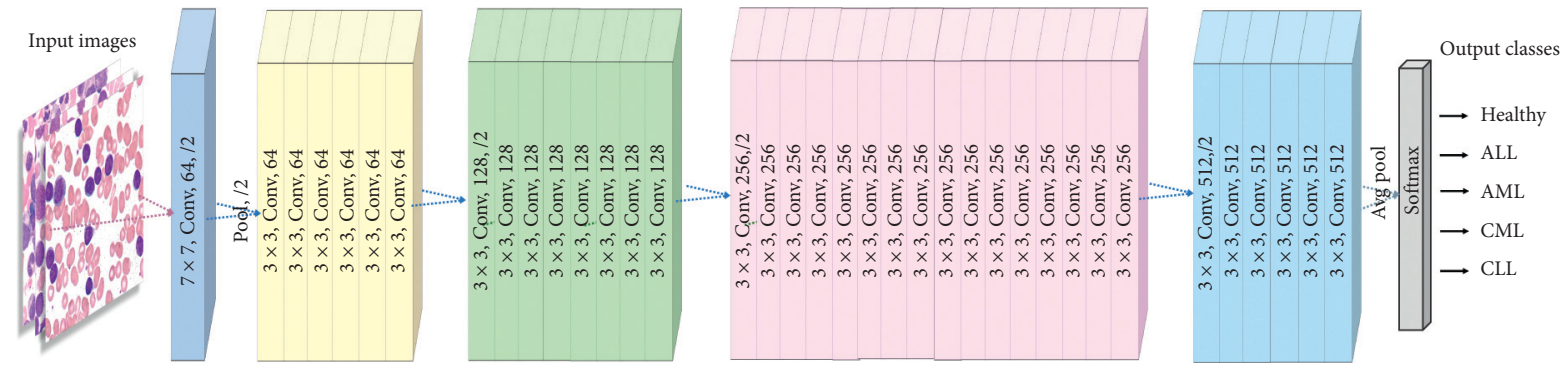

FIgURE 5: General architecture of ResNet-34 (adapted from [28]).

and 512, respectively [28]. The general architecture of RestNet-34 is presented in Figure 5.

3.3.2. DenseNet-121. Dense convolutional networks or DenseNet achieved the best classification results on CIFAR10 and ImageNet datasets [29]. Dense connections are used in the DenseNet architecture, such as ResNet architecture. DenseNet-121 consists of 121 layers. In DenseNet architecture, each layer is connected to all subsequent layers. Thus, each layer receives important features learned by any preceding layers of the network that makes training of the network more efficient [55]. The DenseNet architecture uses fewer parameters than ResNet for the training of the network. Small datasets make the model overfit, while the dense connection solves that overfitting problem [29]. A significant part of DenseNet is a dense block, which is used for enhancing the information flow between layers. It consists of $\mathrm{BN}, \mathrm{ReLU}$, and $3 \times 3$ conv. The particular formula for the dense block is provided in

$$
L_{l}=H_{l}\left(\left[L_{0}, L_{1}, \ldots, L_{l-1}\right]\right),
$$

where $(0,1, \ldots, l-1)$ represents the layers of DenseNet-121 and $\left[L_{0}, L_{1}, \ldots, L_{l-1}\right]$ is the concatenation of feature map obtained from the layers of DenseNet-121. The composite function of $\mathrm{BN}, \mathrm{ReLU}$, and $3 \times 3$ conv. operations on the $l^{\text {th }}$ layer is presented by $\mathrm{Hl}$ (.) [29]. The DenseNet architecture is presented in Figure 6.

Both pre-trained models are implemented with Python using open source fastai [56] and the deep learning library, which makes the implementation of the models simpler. All the experiments are performed on Google Colab [57].

\section{Experimental Results}

In order to evaluate the proposed models, the performance measures used are precision, recall, F1 score, and accuracy. The mathematical description of each of these parameters is given in Table 2. Here, TP is a true positive rate and refers to positive class determined as positive; FP is false positive rate and refers to negative class determined as positive; TN is true negative rate and refers to negative class determined as negative; and FN is false negative rate and refers to positive class determined as negative [58]. However, Recall is the accuracy of prediction for the known leukemia subtype class. Accuracy is the prediction for the known leukemia and nonleukemia subtype classes [59]. Precision is the ratio of correct positive predicted leukemia subtype classes to the predicted positive leukemia subtype classes, while F1 score is the harmonic mean of precision and recall [23].

To show the efficiency of the utilized methods, various parameters such as precision, recall, F1 score, and accuracy 


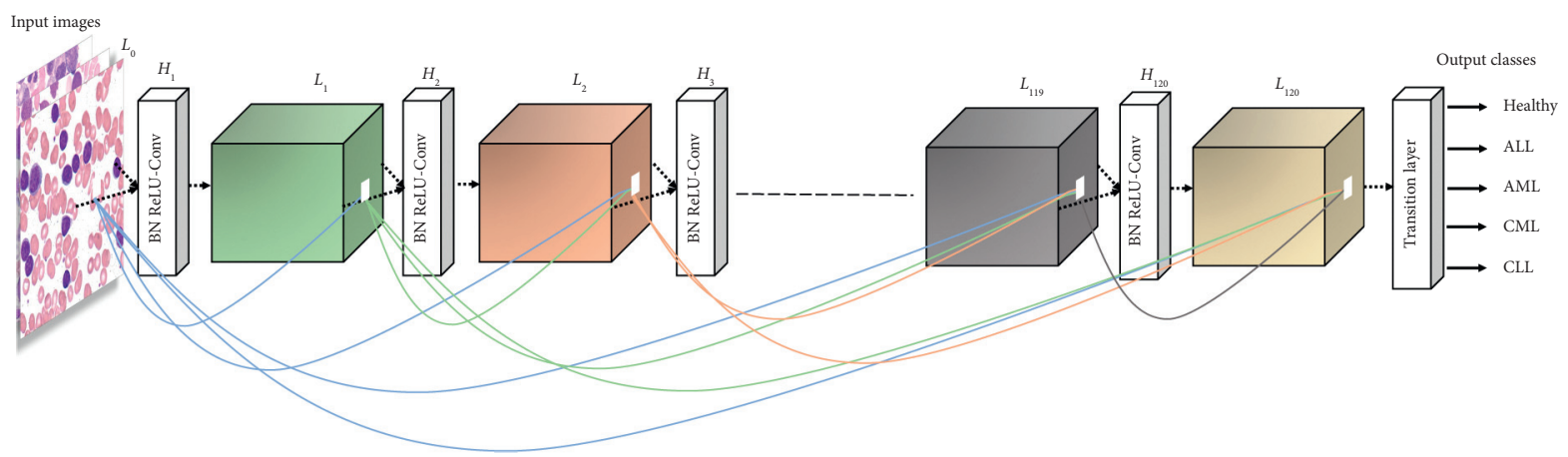

FIgure 6: General architecture of DenseNet-121 (adapted from [29]).

TABLe 2: Performance measures mathematical description.

\begin{tabular}{lc}
\hline Measure & Derivations \\
\hline Accuracy & $\mathrm{ACC}=(\mathrm{TP}+\mathrm{TN}) /(\mathrm{P}+\mathrm{N})$ \\
Precision & $\mathrm{PPV}=\mathrm{TP} /(\mathrm{TP}+\mathrm{FP})$ \\
Recall & $\mathrm{TPR}=\mathrm{TP} /(\mathrm{TP}+\mathrm{FN})$ \\
F1 score & $\mathrm{F} 1=2 \mathrm{TP} /(2 \mathrm{TP}+\mathrm{FP}+\mathrm{FN})$ \\
\hline
\end{tabular}

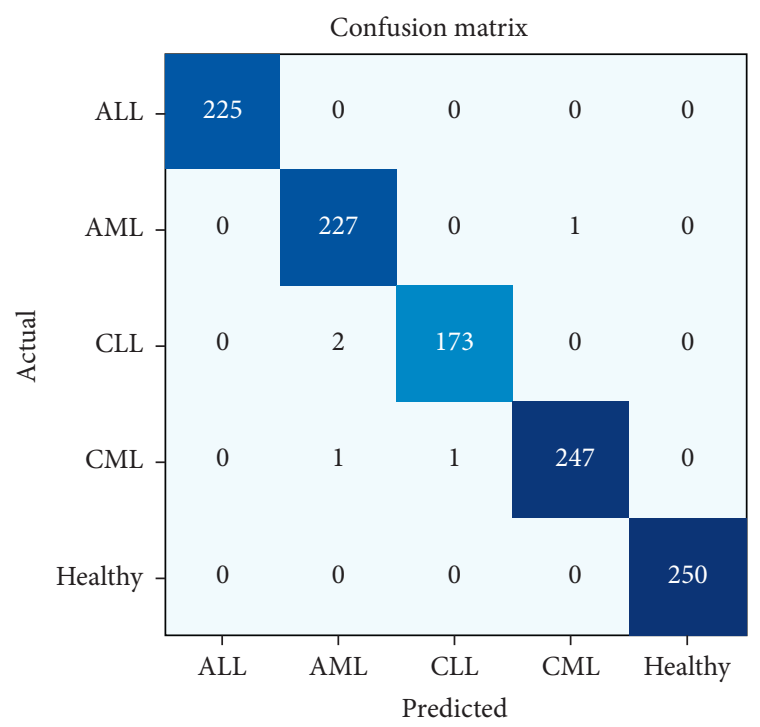

Figure 7: Confusion matrix of ResNet-34 for leukemia subtype classification.

are used. Figures 7 and 8 show the confusion matrix for the classification of subtypes of leukemia using ResNet-34 and DenseNet-121, respectively. It is evident from Figures 7 and 8 that the proposed models predicate very well. Furthermore, Tables 3 and 4 present the accuracy, precision, recall, and F1 score for each type of leukemia on the benchmark dataset, ALL-IDB and ASH image bank.

It is exhibited from Tables 3 and 4 that the ResNet-34 and DenseNet-121 prediction accuracy for ALL and healthy cases is $100 \%$, while precision, recall, and F1 score are also $100 \%$, i.e., 1.0. The prediction accuracy of ResNet-34 for AML is $99.65 \%$, precision is $1.0 \%$, recall is $0.99 \%$, and $\mathrm{F} 1$ score is also $0.99 \%$. In case of CLL, the prediction accuracy of ResNet-34 is $99.73 \%$. However, precision, recall, and F1 score are $0.99 \%$. For CML, the prediction accuracy of
ResNet-34 is $99.73 \%$, precision is $0.99 \%$, recall is $1.0 \%$, and F1 score is $0.99 \%$, whereas in case of DenseNet-121, the prediction accuracy for AML is $99.91 \%$. However, precision, recall, and F1 score are $1.0 \%$. In case of CLL, the prediction accuracy of DenseNet-121 is $99.91 \%$, precision is $1.0 \%$, recall is $0.99 \%$, and F1 score is $1.0 \%$. For CML, the DenseNet- 121 prediction accuracy, precision, recall, and F1 score are $100 \%$.

Moreover, the training and validation loss for ResNet-34 and DenseNet-121 are shown in Figures 9 and 10, respectively. It is depicted from Figures 9 and 10 that the training and validation loss of ResNet-34 and DenseNet-121 are nearly 0 , which means that the accuracy is also near $100 \%$. However, in case of DenseNet-121, the training and validation loss are nearer 0 as compared to ResNet-34. Hence, it is concluded from the results that the prediction performance of ResNet-34 and DenseNet-121 is better for identifying the leukemia subtypes. However, DenseNet-121 seems to supersede ResNet-34. The DenseNet-121 training and validation loss are near 0 when compared to ResNet-34, while for some subtypes identification, i.e., AML, CLL, and CML, DenseNet-121 outperforms ResNet-34. The performance comparison of ResNet-34 and DenseNet-121 with respect to leukemia subtypes identification is shown in Figure 11.

To show their efficiency, a comparison of the proposed models is done with previous approaches, i.e., GA with SVM [49] and CNN [39]. GA with SVM performs the identification of ALL, AML, and healthy samples, while CNN performs the leukemia subtype identification. Nevertheless, it is elucidated in Figure 12 that the utilized models, i.e., ResNet-34 and DenseNet-121, outperform the existing schemes, such as GA with SVM and CNN. It is exhibited from Figure 12 that GA with SVM has $99.50 \%$, CNN has $81.74 \%$, ResNet-34 has $99.56 \%$, and DenseNet-121 has 99.91\% accuracy, respectively. Thus, DenseNet-121 supersedes the other approaches. Previously, numerous machine learning techniques were using the same datasets, i.e., ALLIDB and ASH image bank, for the detection of leukemia and its subtypes. In those studies, the feature extraction and classification methods were used, which require time and effort. However, the proposed models do not need handcrafted feature set for making predictions and save time and efforts. A thorough comparison of the proposed models with the previous approaches with respect to accuracy is presented in Table 5. It is depicted from Table 5 that the 


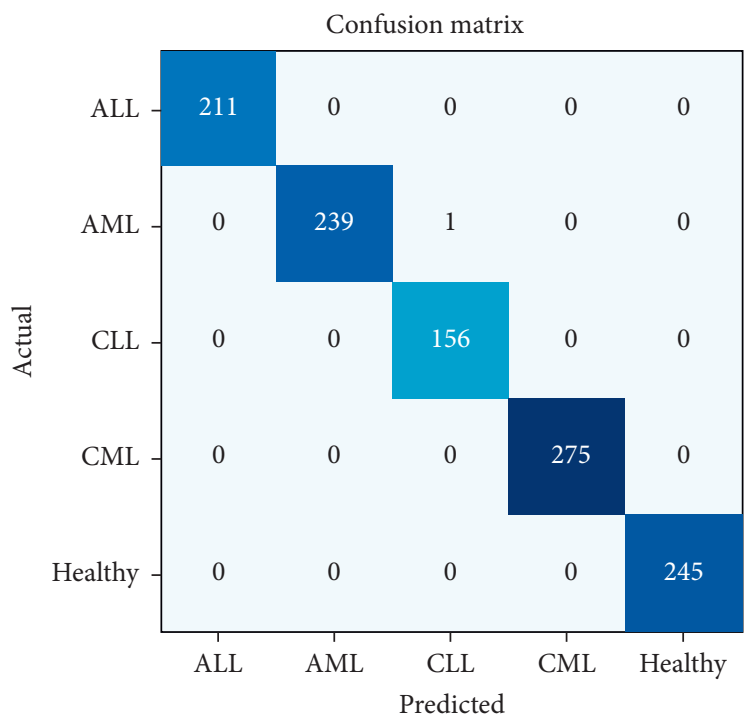

Figure 8: Confusion matrix of DenseNet-121 for leukemia subtype classification.

Table 3: Performance of the ResNet-34 model for leukemia subtype classification.

\begin{tabular}{lcccc}
\hline Leukemia type & Accuracy & Precision & Recall & \\
\hline ALL & 100 & 1.0 & 1.0 & F1 score \\
AML & 99.65 & 1.0 & 0.99 & 0.99 \\
CLL & 99.73 & 0.99 & 0.99 & 0.99 \\
CML & 99.73 & 0.99 & 1.0 & 0.99 \\
Healthy & 100 & 1.0 & 1.0 & 1.0 \\
\hline
\end{tabular}

Table 4: Performance of the DenseNet-121 model for leukemia subtype classification.

\begin{tabular}{lcccc}
\hline Leukemia type & Accuracy & Precision & Recall & \\
\hline ALL & 100 & 1.0 & 1.0 & 1.0 \\
AML & 99.91 & 1.0 & 1.0 & 1.0 \\
CLL & 99.91 & 1.0 & 0.99 & 1.0 \\
CML & 100 & 1.0 & 1.0 & 1.0 \\
Healthy & 100 & 1.0 & 1.0 & 1.0 \\
\hline
\end{tabular}

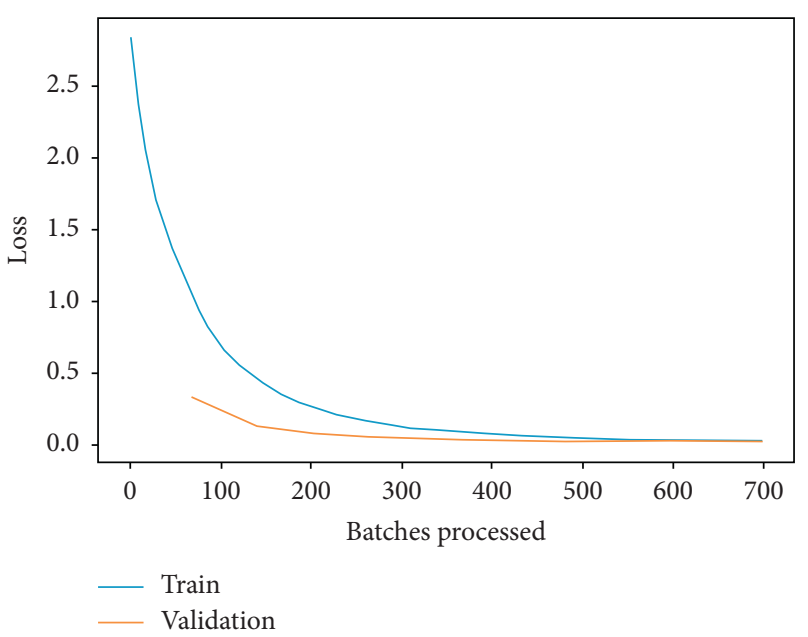

Figure 9: Training and validation loss of ResNet-34. 


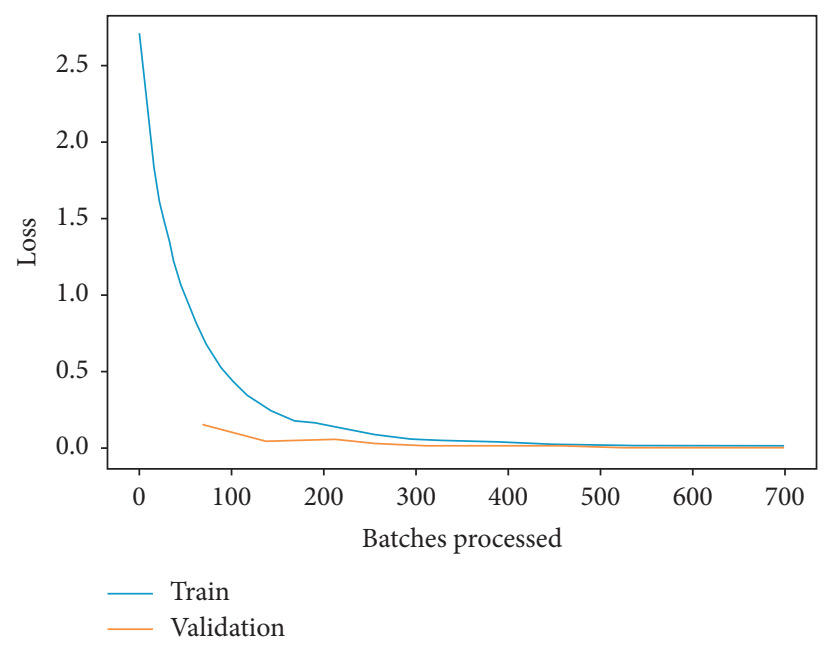

FIgURE 10: Training and validation loss of DenseNet-121.

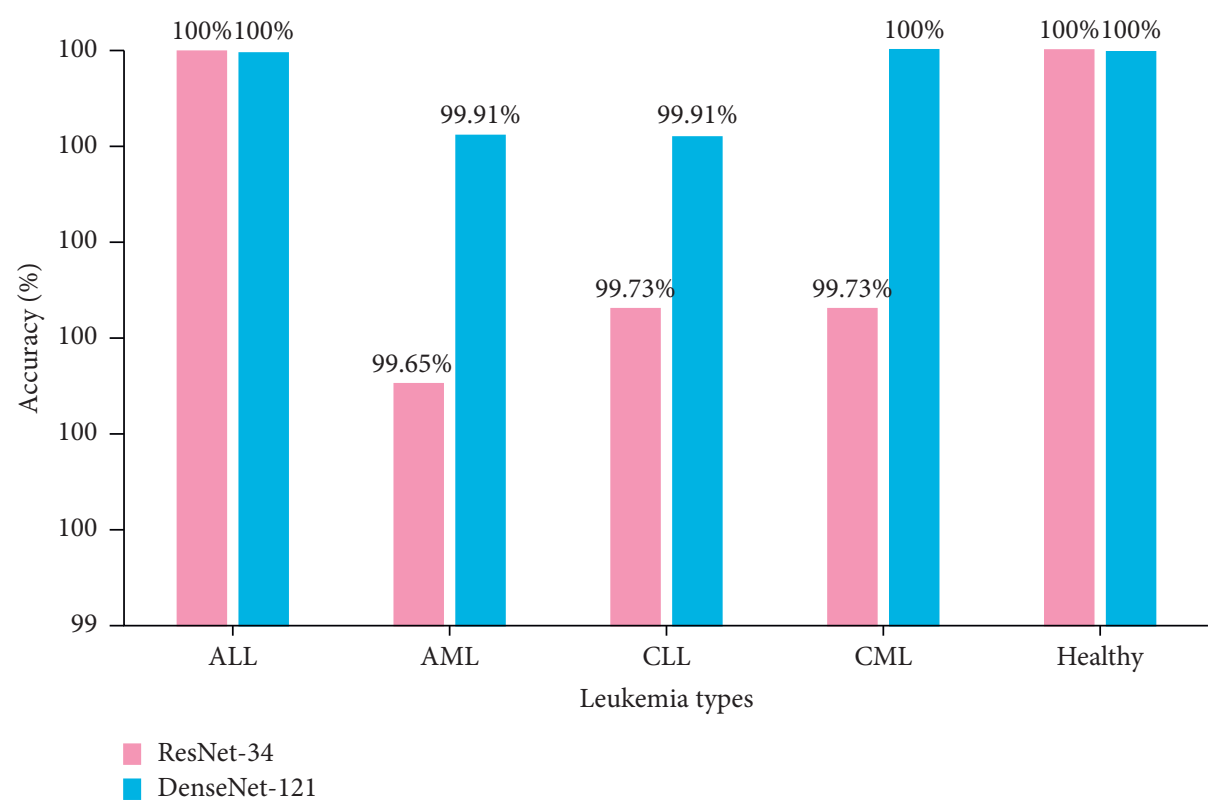

Figure 11: Accuracy comparison of the ResNet-34 and DenseNet-121 for leukemia subtype classification.

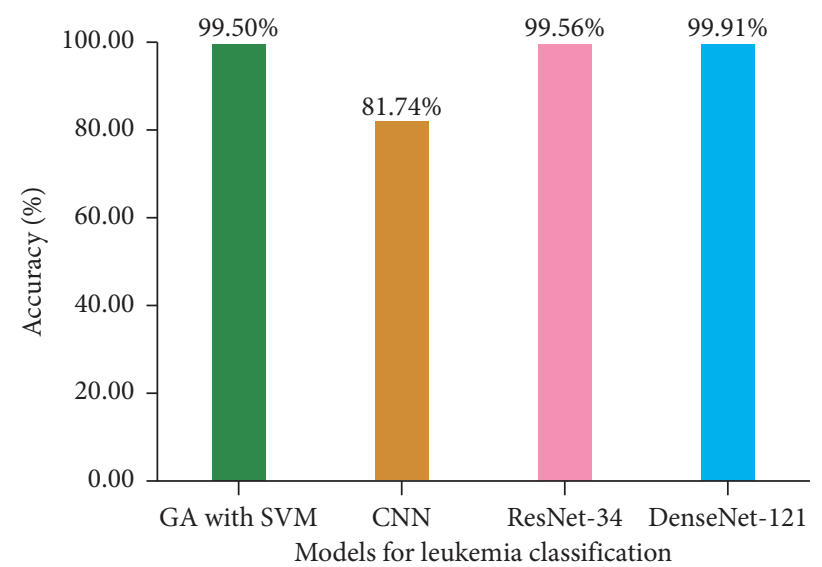

Figure 12: Comparison of the studies on automated detection of subtypes of leukemia. 
TABLE 5: A comparison of the proposed models with the previous approaches for automated detection of leukemia and its subtypes using the same datasets with respect to average accuracy.

\begin{tabular}{|c|c|c|c|c|}
\hline Reference & Dataset & Classification & Classifier & $\begin{array}{c}\text { Accuracy } \\
(\%)\end{array}$ \\
\hline \multirow{10}{*}{ Ahmed et al. [39] } & \multirow{6}{*}{ ALL-IDB } & \multirow{6}{*}{ Leukemia vs healthy } & $\mathrm{CNN}$ & 88.25 \\
\hline & & & Naive Bayes & 69.69 \\
\hline & & & Decision tree & 62.94 \\
\hline & & & KNN & 58.57 \\
\hline & & & SVM & 50.09 \\
\hline & & & $\mathrm{CNN}$ & 81.74 \\
\hline & & & Naive Bayes & 52.68 \\
\hline & ALL-IDB, ASH image bank & Leukemia subtypes & Decision tree & 45.92 \\
\hline & & & KNN & 43.51 \\
\hline & & & SVM & 20.84 \\
\hline \multirow{2}{*}{ Shafique et al. [26] } & \multirow{2}{*}{ ALL-IDB } & $\begin{array}{l}\text { Acute lymphoblastic } \\
\text { leukemia detection }\end{array}$ & AlexNet & 99.50 \\
\hline & & $\begin{array}{l}\text { Subtypes of acute } \\
\text { lymphoblastic leukemia }\end{array}$ & AlexNet & 96.06 \\
\hline \multirow{2}{*}{ Jothi et al. [34] } & \multirow{2}{*}{ ALL-IDB } & Acute lymphoblastic & Jaya, SVM & 99.00 \\
\hline & & leukemia detection & Jaya, decision tree & 98.00 \\
\hline Acharya et al. [40] & ALL-IDB & White blood cells & K-medoids algorithm & 98.60 \\
\hline Mishra et al. [35] & ALL-IDB1 & $\begin{array}{l}\text { Acute lymphoblastic } \\
\text { leukemia detection }\end{array}$ & DOST, PCA, LDA & 99.66 \\
\hline Tuba et al. [41] & ALL-IDB2 & $\begin{array}{l}\text { Acute lymphoblastic } \\
\text { leukemia detection }\end{array}$ & GAO-based methods & 93.84 \\
\hline Al-jaboriy et al. [42] & ALL-IDB1 & $\begin{array}{l}\text { Acute lymphoblastic } \\
\text { leukemia detection }\end{array}$ & GA and ANN & 97.07 \\
\hline Jha et al. [43] & ALL-IDB2 & $\begin{array}{l}\text { Acute lymphoblastic } \\
\text { leukemia detection }\end{array}$ & SCA-based deep CNN & 98.70 \\
\hline Pansombut et al. [44] & \multirow{2}{*}{$\begin{array}{c}\text { ASH image bank, ALL-IDB1 } \\
\text { Heterogeneous database ALL-IDB1, ALL- } \\
\text { IDB2 }\end{array}$} & Lymphoblast cells & CNN-based convnet & 81.74 \\
\hline Vogado et al. [36] & & $\begin{array}{l}\text { Diagnose leukemia } \\
\text { (pathological or not) }\end{array}$ & $\begin{array}{c}\text { Pre-trained CNN with } \\
\text { SVM }\end{array}$ & 99 \\
\hline Thanh et al. [45] & ALL-IDB1 & $\begin{array}{l}\text { Diagnose leukemia } \\
\text { (normal vs abnormal) }\end{array}$ & $\mathrm{CNN}$ & 96.60 \\
\hline Moshavash et al. [46] & $\begin{array}{l}\text { ALL-IDB1, ALL-IDB2, Dr. Juan Bruno Zayas } \\
\text { Alfonso Hospital, Santiago de Cuba }\end{array}$ & $\begin{array}{l}\text { Acute lymphoblastic } \\
\text { leukemia detection }\end{array}$ & $\begin{array}{c}\text { Two ensemble } \\
\text { classifiers with SVM }\end{array}$ & 89.81 \\
\hline $\begin{array}{l}\text { Umamaheswari et al. } \\
\text { [47] }\end{array}$ & ALL-IDB2 & $\begin{array}{c}\text { Acute lymphoblastic } \\
\text { leukemia }\end{array}$ & Customized KNN & 96.25 \\
\hline \multirow[t]{2}{*}{ Agaian et al. [48] } & ALL-IDB1 & $\begin{array}{l}\text { Acute lymphoblastic } \\
\text { leukemia }\end{array}$ & \multirow[t]{2}{*}{$\begin{array}{l}\text { Cell energy feature } \\
\text { with SVM }\end{array}$} & 94.00 \\
\hline & \multirow{3}{*}{ ASH image bank } & ALL & & 97.10 \\
\hline \multirow[t]{2}{*}{ Rawat et al. [49] } & & AML & GA with SVM & 98.50 \\
\hline & & Healthy, AML,ALL & & 99.50 \\
\hline \multirow{2}{*}{ Proposed work } & ALL-IDB, ASH image bank & Healthy, ALL, AML, CLL & ResNet-34 & 99.56 \\
\hline & ALL-IDB, ASH image bank & and CML & DenseNet-121 & 99.91 \\
\hline
\end{tabular}

proposed models outperform the previous approaches with average accuracy of $99.56 \%$ and $99.91 \%$ for ResNet-34 and DenseNet-121, respectively.

\section{Conclusion and Research Directions}

In this study, an IoMT-based framework is proposed for the leukemia subtypes detection. In the proposed framework, an IoT-enabled microscope uploads the blood smear images to the leukemia cloud. The leukemia is diagnosed by using the ResNet-34 or DenseNet-121 models. It is observed that the diagnosing power of ResNet-34 and DenseNet-121 supersedes all the previous approaches. By using data augmentation techniques, ResNet-34 and DenseNet-121 both process large numbers of image patterns. After diagnosis, the result is sent to the doctor's computer where s/he provides medical care on the basis of test report through the IoMT framework. Furthermore, the proposed framework facilitates the patients in pandemics such as COVID-19.

In the future, the dataset can be extended by adding new samples of blood images and utilizing new augmentation techniques to achieve better performances. Furthermore, the proposed IoMT-based framework can be equipped with the functionality of diagnosing the subcategories of each leukemia type. Moreover, the proposed models can also be used to find other abnormalities in the blood.

\section{Data Availability}

The following two datasets have been used in the research: ASH image bank and ALL-IDB publicly available datasets, which have been cited in the paper at proper positions. 


\section{Conflicts of Interest}

The authors declare that they have no conflicts of interest regarding the publication of this work.

\section{Acknowledgments}

This work was supported in part by King Saud University, Saudi Arabia, through research supporting project number RSP-2020/184, and in part by Fundamental Research Funds for Central Universities (No. 2462020YJRC001).

\section{References}

[1] F. M. Malik, H. A. Khattak, A. Almogren, O. Bouachir, I. U. Din, and A. Altameem, "Performance evaluation of data dissemination protocols for connected autonomous vehicles," IEEE Access, vol. 8, pp. 126 896-126906, 2020.

[2] K. A. Awan, I. U. Din, A. Almogren, M. Guizani, and S. Khan, "Stabtrust-a stable and centralized trust-based clustering mechanism for iot enabled vehicular ad-hoc networks," Ieee Access, vol. 8, pp. 21 159-21 177, 2020.

[3] H. A. Khattak, K. Tehreem, A. Almogren, Z. Ameer, I. U. Din, and M. Adnan, "Dynamic pricing in industrial internet of things: blockchain application for energy management in smart cities," Journal of Information Security and Applications, vol. 55, 2020.

[4] K. Haseeb, I. U. Din, A. Almogren, Z. Jan, N. Abbas, and M. Adnan, "Ddr-esc: a distributed and data reliability model for mobile edge-based sensor-cloud," IEEE Access, vol. 8, pp. $185752-185760,2020$.

[5] K. Haseeb, A. Almogren, I. Ud Din, N. Islam, and A. Altameem, "Sasc: secure and authentication-based sensor cloud architecture for intelligent internet of things," Sensors, vol. 20, no. 9, p. 2468, 2020.

[6] W. Ali, I. U. Din, A. Almogren, M. Guizani, and M. Zuair, “A lightweight privacy-aware iot-based metering scheme for smart industrial ecosystems," IEEE Transactions on Industrial Informatics, vol. 20, 2020.

[7] W. Ali, I. U. Din, A. Almogren, and N. Kumar, "Alpha: an anonymous orthogonal code-based privacy preserving scheme for industrial cyber physical systems," IEEE Transactions on Industrial Informatics, vol. 20, 2020.

[8] Z. Ali, M. A. Shah, A. Almogren, I. Ud Din, C. Maple, and H. A. Khattak, "Named data networking for efficient iot-based disaster management in a smart campus," Sustainability, vol. 12, no. 8, 2020.

[9] K. Haseeb, I. U. Din, A. Almogren, N. Islam, and A. Altameem, "Rts: a robust and trusted scheme for iot-based mobile wireless mesh networks," IEEE Access, vol. 8, 2020.

[10] K. Haseeb, I. Ud Din, A. Almogren, and N. Islam, "An energy efficient and secure iot-based wsn framework: an application to smart agriculture," Sensors, vol. 20, no. 7, p. 2081, 2020.

[11] I. U. Din, A. Almogren, M. Guizani, and M. Zuair, "A decade of internet of things: analysis in the light of healthcare applications," IEEE Access, vol. 7, pp. 89 967-89 979, 2019.

[12] H. A. Al Hamid, S. M. M. Rahman, M. S. Hossain, A. Almogren, and A. Alamri, "A security model for preserving the privacy of medical big data in a healthcare cloud using a fog computing facility with pairing-based cryptography," IEEE Access, vol. 5, pp. 22 313-22 328, 2017.

[13] M. G. R. Alam, M. M. Hassan, M. Z. Uddin, A. Almogren, and G. Fortino, "Autonomic computation offloading in mobile edge for iot applications," Future Generation Computer Systems, vol. 90, pp. 149-157, 2019.

[14] I. U. Din, M. Guizani, S. Hassan et al., "The internet of things: a review of enabled technologies and future challenges," Ieee Access, vol. 7, pp. 7606-7640, 2018.

[15] I. U. Din, M. Guizani, J. J. P. C. Rodrigues, S. Hassan, and V. V. Korotaev, "Machine learning in the internet of things: designed techniques for smart cities," Future Generation Computer Systems, vol. 100, pp. 826-843, 2019.

[16] E. K. Wang, C.-M. Chen, M. M. Hassan, and A. Almogren, “A deep learning based medical image segmentation technique in internet-of-medical-things domain," Future Generation Computer Systems, vol. 108, pp. 135-144, 2020.

[17] S. R. Khan, M. Sikandar, A. Almogren, I. Ud Din, A. Guerrieri, and G. Fortino, "Iomt-based computational approach for detecting brain tumor," Future Generation Computer Systems, vol. 109, pp. 360-367, 2020.

[18] N. Islam, Y. Faheem, I. U. Din, M. Talha, M. Guizani, and M. Khalil, "A blockchain-based fog computing framework for activity recognition as an application to e-healthcare services," Future Generation Computer Systems, vol. 100, pp. 569-578, 2019.

[19] K. Janjua, M. A. Shah, A. Almogren, H. A. Khattak, C. Maple, and I. U. Din, "Proactive forensics in iot: privacy-aware logpreservation architecture in fog-enabled-cloud using holochain and containerization technologies," Electronics, vol. 9, no. 7, p. 1172, 2020.

[20] S. U. Khan, N. Islam, Z. Jan, I. U. Din, A. Khan, and Y. Faheem, "An e-health care services framework for the detection and classification of breast cancer in breast cytology images as an iomt application," Future Generation Computer Systems, vol. 98, pp. 286-296, 2019.

[21] K. Haseeb, N. Islam, A. Almogren, and I. U. Din, "Intrusion prevention framework for secure routing in wsn-based mobile internet of things," Ieee Access, vol. 7, pp. 185 496-185 505, 2019.

[22] S. Khan, N. Islam, Z. Jan, I. Ud Din, and J. J. P. C. Rodrigues, "A novel deep learning based framework for the detection and classification of breast cancer using transfer learning," Pattern Recognition Letters, vol. 125, pp. 1-6, 2019.

[23] M. Sikarndar, W. Anwar, A. Almogren, I. Ud Din, and N. Guizani, "Iomt-based association rule mining for the prediction of human protein complexes," IEEE Access, vol. 8, pp. 6226-6237, 2020.

[24] K. A. Awan, I. U. Din, A. Almogren, H. Almajed, I. Mohiuddin, and M. Guizani, "Neurotrust-artificial neural network-based intelligent trust management mechanism for large-scale internet of medical things," IEEE Internet of Things Journal, vol. 8, 2020.

[25] A. Almogren, I. Mohiuddin, I. U. Din, and H. Al Majed, "Ftmiomt: Fuzzy-based trust management for preventing sybil attacks in internet of medical things," IEEE Internet of Things Journal, vol. 8, 2020.

[26] S. Shafique and S. Tehsin, "Acute lymphoblastic leukemia detection and classification of its subtypes using pretrained deep convolutional neural networks," Technology in Cancer Research \& Treatment, vol. 17, 2018.

[27] https://www.cancercenter.com/cancer-types/leukemia/types/ acute-myeloid-leukemia.

[28] K. He, X. Zhang, S. Ren, and J. Sun, "Deep residual learning for image recognition," 2016.

[29] G. Huang, Z. Liu, L. Van Der Maaten, and K. Q. Weinberger, "Densely connected convolutional networks," 2017. 
[30] Y. LeCun, Y. Bengio, and G. Hinton, “Deep learning,” Nature, vol. 521, no. 7553, pp. 436-444, 2015.

[31] H. Mohamed, R. Omar, N. Saeed et al., "Automated detection of white blood cells cancer diseases," 2018.

[32] S. Kumar, S. Mishra, and P. Asthana, "Automated detection of acute leukemia using k-mean clustering algorithm," Advances in Computer and Computational Sciences, vol. 554, pp. 655670, 2018.

[33] R. Sharma and R. Kumar, "A novel approach for the classification of leukemia using artificial bee colony optimization technique and back-propagation neural networks: ICCN 2018," 2019.

[34] J. Ganesan, H. H. Inbarani, A. T. Azar, and K. R. Devi, "Rough set theory with jaya optimization for acute lymphoblastic leukemia classification," Neural Computing and Applications, vol. 55, pp. 1-20, 2018.

[35] S. Mishra, B. Majhi, and P. K. Sa, "Texture feature based classification on microscopic blood smear for acute lymphoblastic leukemia detection," Biomedical Signal Processing and Control, vol. 47, pp. 303-311, 2019.

[36] L. H. S. Vogado, R. M. S. Veras, F. H. D. Araujo, R. R. V. Silva, and K. R. T. Aires, "Leukemia diagnosis in blood slides using transfer learning in cnns and svm for classification," Engineering Applications of Artificial Intelligence, vol. 72, pp. 415-422, 2018.

[37] D. patra and S. satpathi, "Image analysis of blood microscopic images for acute leukemia detection," 2010.

[38] S. A. kareem and H. ariffin, "A robust feature extraction and selection method for the recognition of lymphocytes versus acute lymphoblastic leukemia," 2012.

[39] N. Ahmed, A. Yigit, Z. Isik, and A. Alpkocak, "Identification of leukemia subtypes from microscopic images using convolutional neural network," Diagnostics, vol. 9, no. 3, p. 104, 2019.

[40] V. Acharya and P. Kumar, "Detection of acute lymphoblastic leukemia using image segmentation and data mining algorithms," Medical \& Biological Engineering \& Computing, vol. 57, p. 6, 2019.

[41] M. Tuba and E. Tuba, "Generative adversarial optimization (Goa) for acute lymphocytic leukemia detection," Studies in Informatics and Control, vol. 28, pp. 245-254, 102019.

[42] S. S. Al-jaboriy, N. N. A. Sjarif, S. Chuprat, and W. M. Abduallah, "Acute lymphoblastic leukemia segmentation using local pixel information," Pattern Recognition Letters, vol. 125, pp. 85-90, 2019.

[43] K. K. Jha and H. S. Dutta, "Mutual information based hybrid model and deep learning for acute lymphocytic leukemia detection in single cell blood smear images," Computer Methods and Programs in Biomedicine, vol. 179, 2019.

[44] T. Pansombut, S. Wikaisuksakul, K. Khongkraphan, and A. Phon-on, "Convolutional neural networks for recognition of lymphoblast cell images," Computational Intelligence and Neuroscience, vol. 2019, 2019.

[45] T. T. P. Thanh, C. Vununu, C. Vununu, S. Atoev, S.-H. Lee, and K.-R. Kwon, "Leukemia blood cell image classification using convolutional neural network," International Journal of Computer Theory and Engineering, vol. 10, no. 2, pp. 54-58, 2018.

[46] Z. Moshavash, H. Danyali, and M. S. Helfroush, "An automatic and robust decision support system for accurate acute leukemia diagnosis from blood microscopic images," Journal of Digital Imaging, vol. 31, no. 5, pp. 702-717, 2018.

[47] D. Umamaheswari and S. Geetha, "A framework for efficient recognition and classification of acute lymphoblastic leukemia with a novel customized-knn classifier," Journal of Computing and Information Technology, vol. 26, no. 2, pp. 131-140, 2018.

[48] S. Agaian, M. Madhukar, and A. T. Chronopoulos, "A new acute leukaemia-automated classification system," Computer Methods in Biomechanics and Biomedical Engineering: Imaging \& Visualization, vol. 6, no. 3, pp. 303-314, 2018.

[49] J. Rawat, A. Singh, B. Hs, J. Virmani, and J. S. Devgun, "Computer assisted classification framework for prediction of acute lymphoblastic and acute myeloblastic leukemia," Biocybernetics and Biomedical Engineering, vol. 37, no. 4, pp. 637-654, 2017.

[50] ASH Image Bank, "The american society of hematology," 2017.

[51] R. D. Labati, V. Piuri, and F. Scotti, "All-idb: the acute lymphoblastic leukemia image database for image processing," 2011.

[52] ALL-IDB, "Acute lymphoblastic leukemia image database processing," 2017.

[53] K. Simonyan and A. Zisserman, "Very deep convolutional networks for large-scale image recognition," 2014.

[54] A. Krizhevsky, I. Sutskever, and G. E. Hinton, "Imagenet classification with deep convolutional neural networks," 2012.

[55] L. Li, J. Liang, M. Weng, and H. Zhu, "A multiple-feature reuse network to extract buildings from remote sensing imagery," Remote Sensing, vol. 10, p. 1350, 2018.

[56] Fastai, "The fastai deep learning library," 2018.

[57] Colab, "Google colaboratory," 2018.

[58] E. Urtnasan, J.-U. Park, and K.-J. Lee, "Multiclass classification of obstructive sleep apnea/hypopnea based on a convolutional neural network from a single-lead electrocardiogram," 2018.

[59] Z.-C. Li, Y.-H. Lai, L.-L. Chen, X. Zhou, Z. Dai, and X.-Y. Zou, "Identification of human protein complexes from local subgraphs of protein-protein interaction network based on random forest with topological structure features," Analytica Chimica Acta, vol. 718, pp. 32-41, 2012. 